\title{
DISTRIBUIÇÃO NATURAL DE ESPÉCIES ARBÓREAS EM ÁREAS COM DIFERENTES NÍVEIS DE ANTROPIZAÇÃO. ATRIBUTOS FÍSICOS DO SOLO
}

\author{
Hemerson Fernandes Calgaro ${ }^{1}$, José Cambuim ${ }^{2}$, Alexandre Marques da Silva ${ }^{3}$, Marlene \\ Cristina Alves ${ }^{4}$, Salatiér Buzetti ${ }^{4}$, Marcela Aparecida de Moraes ${ }^{5}$, Sérgio Luís de Carvalho ${ }^{6}$, \\ Luiz Paulo Montenegro de Miranda ${ }^{5}$, Mario Luiz Teixeira de Moraes ${ }^{7}$
}

\footnotetext{
${ }^{1}$ Assistente Agropecuário da Secretaria de Agricultura e Abastecimento do Estado de São Paulo.

2 Administrador, Auxiliar Agropecuário na Fazenda de Ensino, Pesquisa e Extensão - Doutorando do Programa de Pós-Graduação em Agronomia da UNESP Campus de Ilha Solteira (SP).

${ }^{3}$ Geógrafo, Técnico Agropecuário do Departamento de Fitotecnia, Tecnologia de Alimentos e Sócio Economia - Doutorando do Programa de Pós-Graduação em Agronomia da UNESP Campus de Ilha Solteira (SP).

${ }^{4}$ Prof. Titular do Departamento de Fitossanidade, Engenharia Rural e Solos da UNESP Campus de Ilha Solteira (SP).

${ }^{5}$ Doutorandos do Programa de Pós-Graduação em Agronomia da UNESP Campus de Ilha Solteira (SP).

${ }^{6}$ Prof. Adjunto do Departamento de Biologia e Zootecnia da UNESP Campus de Ilha Solteira (SP).

${ }^{7}$ Prof. Titular do Departamento de Fitotecnia, Tecnologia de Alimentos e Sócio Economia da UNESP Campus de Ilha Solteira (SP) - Av. Brasil 56, Centro - CEP 15.385-000, Ilha Solteira (SP), Brasil.
}

RESUMO: Com o surgimento de áreas degradadas, os atributos físicos do solo e os caracteres silviculturais, passaram a ter importância quanto ao entendimento do processo sucessional das espécies arbóreas e no planejamento da recomposição. Objetivou-se avaliar a distribuição natural de espécies arbóreas em áreas com diferentes níveis de antropização, relacionando-os aos atributos físicos do solo. O projeto foi conduzido no município de Selvíria - MS. Foram demarcadas 64 parcelas, onde coletaram-se amostras de solo em quatro profundidades para análise física do solo e avaliaram-se os caracteres silviculturais, como altura, diâmetro a altura do peito (DAP) e forma. Verificaram-se diferenças na ocupação florestal, diversidade de espécies e desenvolvimento dos indivíduos arbóreos, em função do nível de antropização, estado de conservação das áreas e dos atributos físicos do solo. Quanto mais isolada a área, maior a ocupação florestal, haja vista a área pouco antropizada (APA - revegetada) e a Reserva legal, que apresentaram valores de número de indivíduos e número de espécies por parcela, superiores às demais áreas. A mata ciliar apresenta valores de área basal, altura e forma, superiores às demais áreas, entretanto, os valores de macroporosidade do solo foram inferiores, quando comparados às demais áreas. Em função da presença de vegetação e das condições dos atributos físicos do solo, nas diversas áreas estudadas, seis espécies arbóreas têm potencial para um programa de conservação genética in situ: Astronium fraxinifolium, Terminalia argentea, Curatella americana, Cupania vernalis, Qualea jundiahy e Andira cuyabensis. Entre as espécies, a $C$. americana pode ser considerada como espécie nucleadora.

Palavras-chave: Cerrado. Porosidade do solo. Manejo do solo. Área degradada.

Cultura Agronômica, Ilha Solteira, v.24, n.3, p.327-344, 2015 


\section{NATURAL DISTRIBUTION OF TREE SPECIES IN AREAS WITH DIFFERENT LEVELS OF ANTHROPOGENIC. SOIL PHYSICAL CHARACTERISTICS}

ABSTRACT: With the emergence of degraded areas, the soil physical attributes and silvicultural characters, have been given importance in understanding the succession process of tree species and in planning the restoration. This study aimed to evaluate the natural distribution of tree species in areas with different levels of human disturbance, relating them to the soil physical properties. The project was conducted in Selvíria - MS. 64 plots were demarcated, which is collected soil samples in four depths for physical soil analysis and evaluated the silvicultural parameters such as height, diameter at breast height (DBH) and form. There were differences in forest occupation, species diversity and development of individual trees, according to the anthropic level, conservation status of areas and soil physical properties. The more isolated the area, the greater the forest occupation, given the little disturbed areas (APA - revegetated) and the Legal reserve, which showed the number of values of individuals and number of species per plot, higher than other areas. The riparian vegetation has basal area, height and shape, superior to the other areas, however, soil macroporosity values were lower when compared to other areas. Depending on the presence of vegetation and the conditions of soil physical properties in various areas studied, six tree species have potential for genetic conservation program in situ: Astronium fraxinifolium, Terminalia argentea, American Curatella, Cupania vernalis, Qualea jundiahy and Andira cuyabensis. Among species, the $C$. americana nucleation can be considered as species.

Key words: Savanna. Soil porosity. Soil management. Degraded area.

\section{INTRODUÇÃO}

A Mata Atlântica, o bioma brasileiro mais devastado, perdeu $751 \mathrm{mil} \mathrm{km}^{2}$, no Cerrado, a transformação da paisagem foi muito mais rápida, em 40 anos perdeu 800 mil $\mathrm{km}^{2}$ (ZORZETTO, 2008), dando lugar a abertura de novas fronteiras agrícolas e atraindo a agroindústria nacional (SILVA, 2007). A distribuição e a manutenção das diferentes fitofisionomias do Bioma Cerrado estão relacionadas a fatores edáficos, topográficos, ocorrência de fogo e perturbações antrópicas (EITEN, 1982).

Com relação as Áreas de Preservação Permanente, estas estão entre as interfaces mais mal trabalhadas dentro da legislação ambiental federal (ARAUJO, 2002). Estas formações apresentam grandes variações em sua composição florística e estrutural, sendo relacionadas com as características intrínsecas da área como relevo, solo, largura da faixa ciliar e do curso d'água, flutuação do lençol freático e histórico de perturbações (DURIGAN et al., 2000).

A Reserva legal localiza-se no interior de uma propriedade ou posse rural, de domínio público ou privado, destinada ao uso sustentável dos recursos naturais, à conservação e reabilitação dos processos ecológicos, à conservação da biodiversidade e ao abrigo e proteção de fauna e flora nativas, segundo o Código Florestal, MP ${ }^{\circ}$ 2.166/67, de 
Em áreas degradadas, os principais fatores que limitam o restabelecimento de uma floresta são, a compactação; empobrecimento do solo; ausência e inutilização do banco de sementes; distância de fontes de propágulos; condições inadequadas à germinação de sementes e reincidência de incêndios (SIQUEIRA, 2002). A reabilitação de áreas degradadas não consiste de ações isoladas, e sim de um conjunto de atividades que têm por objetivo recompor a paisagem perturbada, sendo raras as pesquisas que procuram avaliar a qualidade do solo sob o enfoque de degradação (ALVES; SOUZA, 2008).

A redução de áreas ocupadas por vegetação nativa tem reduzido a biodiversidade e empobrecido os recursos genéticos (MYERS et al., 2000). A restauração destas áreas tem como atividade básica, a conservação genética in situ, refazendo comunidades e estabelecendo corredores entre fragmentos vegetacionais (REIS et al., 2003). O fluxo gênico via pólen e sementes e o status sucessional são os fatores principais que determinam a estrutura genética de populações naturais (KAGEYAMA et al., 2001).

O presente trabalho teve como objetivo avaliar os caracteres silviculturais de indivíduos arbóreos encontrados em áreas perturbadas pela ação antropogênica e áreas preservadas, relacionando-os aos atributos físicos do solo.

\section{MATERIAL E MÉTODOS}

O experimento foi conduzido na Fazenda de Ensino, Pesquisa e Extensão - FEPE, da Faculdade de Engenharia de Ilha Solteira - UNESP, localizada no município de Selvíria (MS), em um Latossolo Vermelho Distrófico típico textura média, "A" moderado, hipodistrófico, álico, caulinítico, hipoférrico, muito profundo, fortemente ácido (DEMATTÊ, 1980; EMBRAPA, 2006).

Originalmente, as áreas degradadas da FEPE apresentavam como cobertura vegetal o Cerrado stricto sensu, porém no final da década de 60, com a construção da Usina Hidrelétrica de Ilha Solteira, houve no local e entorno, desmatamento, sendo retirada uma camada de solo que foi utilizada nas obras de aterro da barragem. Estas áreas, designadas, como "área de empréstimo", tiveram corte e subtração de solo, variando de 8 a $12 \mathrm{~m}$ de profundidade. A "área de empréstimo" pode ser considerada área degradada, uma vez que dela foram retirados os horizontes superficiais do solo (RODRIGUES et al., 2007). Pequenas áreas estão em processo de regeneração natural, mas em sua maior extensão, o subsolo permanece exposto, desprovido de cobertura vegetal e com presença marcante de acentuados processos erosivos (RODRIGUES; MALTONI, 2002; RODRIGUES et al., 2003).

A área em estudo teve por base um transecto iniciando na Reserva Legal da FEPE e finalizando na mata ciliar do córrego da Véstia, atravessando a área degradada (Figura 1), com extensão total de aproximadamente $2.800 \mathrm{~m}$.

Cultura Agronômica, Ilha Solteira, v.24, n.3, p.327-344, 2015 
Ao todo foram demarcadas e georeferenciadas 64 parcelas, sendo que, cada uma detinha área igual a $100 \mathrm{~m}^{2}(10 \times 10 \mathrm{~m})$, mantendo-se equidistância média de $50 \mathrm{~m}$, somando área total de $6.400 \mathrm{~m}^{2}(0,64$ ha).

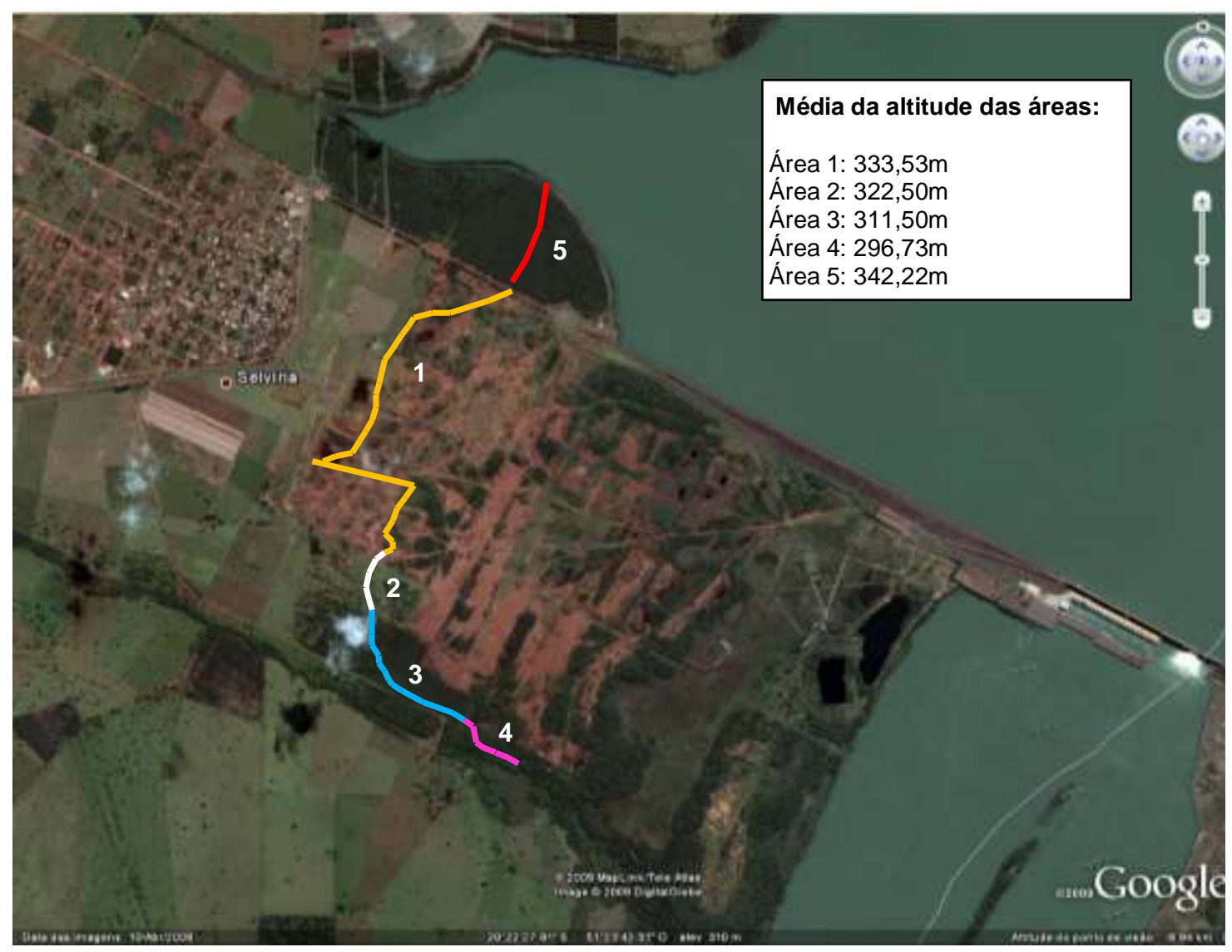

Figura 1. Imagem aérea datada de 2006, obtida pelo software Google Earth, com identificação do transecto, onde foram alocadas as parcelas. 1 - Área altamente antropizada (AAA); 2 - Área medianamente antropizada (AMA); 3 - Área pouco antropizada (APA revegetada); 4 - Mata ciliar; 5 - Reserva legal.

Desta forma, com base no nível de antropização foram identificadas cinco áreas distintas: (1) Área considerada altamente antropizada (AAA), com 29 parcelas; (2) Área considerada medianamente antropizada (AMA), com 5 parcelas; (3) Área considerada pouco antropizada e revegetada espontaneamente com indivíduos arbóreos, em estágio inicial de regeneração (APA - revegetada), com 15 parcelas; (4) na mata ciliar do córrego da Véstia (MC) com 6 parcelas e (5) Reserva legal (RL), com 9 parcelas.

As áreas se caracterizam por: área 1 pelo subsolo exposto desde 1969 (ALVES; SOUZA, 2008) pouca vegetação, tanto rasteira como arbórea e evidentes processos erosivos; área 2 pela presença homogênea de gramíneas, sob um regime de pastejo extensivo; área 3 por estar revegetada, naturalmente, com indivíduos arbóreos nativos, dossel semi-fechado e estágio inicial de regeneração; área 4 pela presença de serapilheira, ausência de gramíneas, árvores de porte alto, grande número de indivíduos regenerantes e relato de que há 12 anos, serviu como área de pastejo, inclusive com trânsito de trator com roçadeira de arrasto; área 5 apresenta grande quantidade de serapilheira, dossel fechado, 
baixa infestação de plantas daninhas e grande número de indivíduos arbóreos e diversidade de espécies.

Os caracteres silviculturais avaliados foram: altura total da planta (ALT) em metros, utilizando aparelho dendrométrico (Vertex III e Transponder T3) e diâmetro a altura do peito (DAP) em centímetros, mensurando-se o perímetro com uma fita métrica, com vistas a minimizar as irregularidades presentes nos troncos dos indivíduos arbóreos. A forma do tronco das árvores teve como base o trabalho de Guerra (2008) em que foram estabelecidas notas para fuste a 2,20 m (medida de interesse comercial), onde a forma do tronco (FOR) variou numa escala de 1 a 5, tanto para Bifurcação (B) como para Retidão (R), sendo que a nota final foi dada, utilizando-se a expressão:

$$
\text { FOR }=\frac{B+R}{2}
$$

Avaliaram-se todas as espécies arbóreas com diâmetro a altura do peito igual ou superior a $0,06 \mathrm{~m}$ e identificaram-se com base na literatura.

Coletou-se em 2009, no centro geométrico de cada parcela, para fins de analisar os atributos físicos do solo, uma amostra indeformada com anel volumétrico de bordo inferior cortante e capacidade interna de $100 \mathrm{~cm} 3$, nas profundidades de $0-0,10 \mathrm{~m} ; 0,10-0,20 \mathrm{~m}$; 0,20-0,30 m e 0,30-0,40 m. Os atributos físicos do solo analisados foram: densidade do solo $\left(\mathrm{kg} \mathrm{dm}^{-3}\right)$ pelo método do anel volumétrico e microporosidade $\left(\mathrm{m}^{3} \mathrm{~m}^{-3}\right)$ em mesa de tensão com coluna de água de $60 \mathrm{~cm}$, de acordo com a metodologia descrita em Embrapa (1997). A porosidade total $\left(\mathrm{m}^{3} \mathrm{~m}^{-3}\right)$ foi determinada saturando-se o solo e determinando-se o volume de água necessário para a saturação e a macroporosidade $\left(\mathrm{m}^{3} \mathrm{~m}^{-3}\right)$ determinada por diferença entre a porosidade total e microporosidade.

Neste estudo foi utilizado delineamento em blocos casualizados desbalanceados na análise dos caracteres silviculturais (Tabela 1) e atributos físicos do solo, para cada uma das profundidades estudadas. $\mathrm{Na}$ análise dos dados foi utilizado o programa estatístico SAS (1999). Os dados de média apresentados nas Tabelas 3 e 4 são os originais, os do teste $\mathrm{F}$ e CV foram obtidos com base na seguinte transformação: $\sqrt{ }(x+0,5)$.

Tabela 1. Esquema da análise de variância em blocos casualizados desbalanceados.

\begin{tabular}{lccc}
\hline Fonte de Variação & GL & QM & F \\
\hline Áreas & $\mathrm{a}-1$ & $\mathrm{Q}_{1}$ & $\mathrm{Q}_{1} / \mathrm{Q}_{3}$ \\
Parcelas & $\mathrm{p}-1$ & $\mathrm{Q}_{2}$ & $\mathrm{Q}_{2} / \mathrm{Q}_{3}$ \\
Erro & $(\mathrm{a}-1)(\mathrm{p}-1)$ & $\mathrm{Q}_{3}$ & \\
\hline Total & $\mathbf{n}-(\mathbf{p}+\mathbf{a}-\mathbf{1})$ & & \\
\hline
\end{tabular}

\section{RESULTADOS E DISCUSSÃO}

Ao todo foram identificadas 98 espécies nos $6400 \mathrm{~m}^{2}$ (Tabela 2) que, segundo a literatura foram sub-divididas em 54 espécies arbóreas oriundas do Cerrado e pertencentes a 34 Famílias; 26 espécies da Floresta Estacional Semidecidual (FES) e pertencentes a 18 Famílias; 9 espécies de transição entre Cerrado e FES, contidas em 8 Famílias; 8 espécies da Mata Pluvial Atlântica e pertencentes a 6 Famílias; e uma N.I. (não identificada).

Cultura Agronômica, Ilha Solteira, v.24, n.3, p.327-344, 2015 
Tabela 2. Relação de espécies vegetais identificadas e classificadas conforme seu bioma de origem (Cerrado - CER; Floresta Estacional Semi-decidual - FES; Vegetação de Transição - CER/FES; Floresta Pluvial Atlântica - FPA), bem como as áreas onde foram encontradas (AAA: 1, AMA: 2, APA - revegetada: 3, Mata ciliar - MC: 4, Reserva legal - RL: 5). Selvíria - MS, 2010.

\begin{tabular}{|c|c|c|c|c|c|c|}
\hline N. & Nome científico & Família & Área & Nome científico & Família & Área \\
\hline \multicolumn{7}{|c|}{ C E R } \\
\hline 1 & Astronium fraxinifolium & Anacardiaceae & $1,2,3,5$ & 28 Plathymenia reticulata & Leg.Mimosoideae & 2,5 \\
\hline 2 & Anacardium humile* & Anacardiaceae & 5 & 29 Stryphnodendron adstringens & Leg.Mimosoideae & 1,3 \\
\hline 3 & Annona crassiflora* & Annonaceae & 2,3 & 30 Acosmium dasycarpum* & Leg.Papilionoideae & 2 \\
\hline 4 & Xylopia aromática & Annonaceae & 1,5 & 31 Dipteryx alata* & Leg.Papilionoideae & 3 \\
\hline 5 & Annona pygmaea* & Annonaceae & $1,3,5$ & 32 Machaerium hirtum & Leg.Papilionoideae & 3 \\
\hline 6 & Annona coriacea* & Annonaceae & 1,3 & 33 Bowdichia virgilioides & Leg.Papilionoideae & 5 \\
\hline 7 & Iiex cerasifolia & Aquifoliaceae & 5 & 34 Byrsonima verbascifolia & Malpighiaceae & $1,2,5$ \\
\hline 8 & Eremanthus erythropappus & Asteraceae & 1 & 35 Heteropterys byrsonimifolia & Malpighiaceae & 1,3 \\
\hline 9 & Tabebuia ochracea & Bignoniaceae & 3,4 & 36 Eriotheca pubescens & Malvaceae & 1,5 \\
\hline 10 & Tabebuia aurea & Bignoniaceae & 1 & 37 Miconia burchellii & Melastomataceae & 5 \\
\hline 11 & Cybistax antisyphilitica* & Bignoniaceae & 1,3 & 38 Mimosa claussenii* & Mimosoideae & $1,2,3,5$ \\
\hline 12 & Anemopaegma arvense* & Bignoniaceae & 1 & 39 Brosimum gaudichaudii & Moraceae & 1 \\
\hline 13 & Pseudobombax longiflorum* & Bombacaceae & 3,5 & 40 Campomanesia sessiliflora* & Myrtaceae & 1 \\
\hline 14 & Caryocar brasiliense & Caryocaraceae & 1,3 & 41 Andira paniculata* & Papilionoideae & 1 \\
\hline 15 & Hirtella gracilipes* & Chrysobalanaceae & 2 & 42 Roupala montana & Proteaceae & 3 \\
\hline 16 & Terminalia argentea & Combretaceae & $1,2,3,5$ & 43 Tocoyena formosa & Rubiaceae & 3 \\
\hline 17 & Gochnatia polymorpha & Compositae & 1 & 44 Alibertia sessiis & Rubiaceae & $2,3,5$ \\
\hline 18 & Connarus suberosus & Connaraceae & 1 & 45 Magonia pubescens & Sapindaceae & 3 \\
\hline 19 & Rourea induta* & Connaraceae & 1,5 & 46 Simarouba versicolor* & Simaroubaceae & 2 \\
\hline 20 & Curatella americana & Dilleniaceae & $1,2,3,5$ & 47 Solanum lycocarpum* & Solanaceae & 1 \\
\hline 21 & Davilla elliptica* & Dilleniaceae & 1 & 48 Solanum paniculatum* & Solanaceae & 1 \\
\hline 22 & Diospyros hispida & Ebenaceae & 1 & 49 Styrax ferrugineus $*$ & Styracaceae & $1,3,5$ \\
\hline 23 & Casearia sylvestris & Flacourtiaceae & 1 & 50 Qualea grandiflora & Vochysiaceae & 1,5 \\
\hline 24 & Kielmeyera variabilis & Guttiferae & 1 & 51 Vochysia cinnamomea & Vochysiaceae & $3,4,5$ \\
\hline 25 & Dimorphandra mollis & Leg.Caesalpinaceae & 3 & 52 Salvertia convallariaeodora* & Vochysiaceae & 1 \\
\hline 26 & Bauhinia longifolia & Leg.Caesalpinioi & 3 & 53 Qualea jundiahy & Vochysiadeae & $1,2,3,5$ \\
\hline 27 & Sclerolobium aureum* & Leg.Caesalpinioideae & 5 & & & \\
\hline \multicolumn{7}{|c|}{ F E S } \\
\hline 1 & Myracrodruon urundeuva & Anarcadiaceae & $1,3,4$ & 14 Albizia niopoides & Mimosaceae & 4 \\
\hline 2 & Tapirira guianensis & Anarcadiaceae & 3,5 & 15 Campomanesia xanthocarpa* & Myrtaceae & $1,2,3,5$ \\
\hline 3 & Tabebuia roseo alba & Bignoniaceae & 3,4 & 16 Eugenia pyriformis* & Myrtaceae & 2,3 \\
\hline & Zeyheria tuberculosa* & Bignoniaceae & 3 & 17 Eugenia uniflora & Myrtaceae & 1 \\
\hline & Cordia ecalyculata* & Boraginaceae & 3,4 & 18 Myrcia selloi & Myrtaceae & 3 \\
\hline 6 & Cordia trichotoma & Boraginaceae & 3 & 19 Colubrina glandulosa & Rhamnaceae & 5 \\
\hline & Casearia gossypiosperma & Flacourtiaceae & 3 & 20 Rhamnidium elaeocarpus & Rhamnaceae & 3,4 \\
\hline & Ocotea velutina & Lauraceae & 5 & 21 Genipa americana & Rubiaceae & 3 \\
\hline & Acacia polyphylla & Leg.Mimosaceae & 1,4 & 22 Zanthoxylum riedelianum & Rutaceae & 4 \\
\hline 10 & Albizia polycephala & Leg.Mimosoideae & 3,4 & 23 Cupania vernalis & Sapindaceae & $2,3,4,5$ \\
\hline 11 & Anadenanthera peregrina & Leg.Mimosoideae & 3,5 & 24 Dilodendron bipinatum & Sapindaceae & 3 \\
\hline 12 & Machaerium villosum & Leg.Papilionoideae & 3,4 & 25 Guazuma ulmifolia & Sterculiaceae & 1 \\
\hline & Hymenaea courbaril* & Leg.Caesalpinoideae & 5 & 26 Luehea candicans & Tiliaceae & 3 \\
\hline \multicolumn{7}{|c|}{ CER/F E S } \\
\hline & Aspidosperma subincanum & Apocynaceae & 3,5 & 6 Diptychandra aurantiaca & Leg.Caesalpinoideae & 3 \\
\hline & Jacaranda cuspidifolia & Bignoniaceae & 3 & 7 Andira cuyabensis & Leg. Papilionoideae & $1,2,3,5$ \\
\hline & Cordia sellowiana & Boraginaceae & 4 & 8 Copaifera langsdorffii & Leg.Caesalpinoideae & 3 \\
\hline & Mabea fistulifera* & Euphorbiaceae & 1,5 & 9 Pouteria ramiflora* & Sapotaceae & 5 \\
\hline & Salacia elliptica* & Hippocrateaceae & 1,2 & & & \\
\hline \multicolumn{7}{|c|}{ F P A } \\
\hline & Annona cacans $*$ & Annonaceae & 2 & 5 Psidium Laurifolium* & Myrtaceae & 1 \\
\hline & Tabebuia heptaphylla & Bignoniaceae & 3 & 6 Psidium guajava* & Myrtaceae & 1 \\
\hline & Inga edulis* $*$ & Leg.Mimosoideae & 4 & 7 Bathysa australis & Rubiaceae & 5 \\
\hline & Inga sp. & Leg.Mimosoideae & 4 & 8 Metrodorea nigra* & Rutaceae & 3,5 \\
\hline 1 & não identificada & não identificada & 5 & & & \\
\hline
\end{tabular}

Cultura Agronômica, Ilha Solteira, v.24, n.3, p.327-344, 2015 
O número total de indivíduos identificados foi de 3.113. Entretanto, deste total somente 545 foram avaliados por apresentarem diâmetro a altura do peito (DAP) igual ou superior a 0,06 m. Verificou-se um predomínio de espécies do bioma Cerrado, contudo, a presença de espécies de outro bioma caracteriza uma área de transição (Ecótono) entre os biomas Cerrado e Mata Atlântica na região de estudo.

Quanto a altura, a MC foi onde se obteve maior valor médio, superando estatisticamente todas as áreas. A AAA apresentou o menor valor, provavelmente por estar inserida em local cuja degradação se deu de forma mais intensa. Já as demais áreas apresentaram semelhança estatística (Tabela 3).

Tabela 3. Valores médios de Altura, DAP, Forma, Área basal (G), Número de indivíduos por parcela (I) e Número de espécies por parcela (S), atribuídos aos indivíduos arbóreos presentes nas parcelas avaliadas, distribuídas nas diferentes áreas.

\begin{tabular}{lcccccc}
\hline \multicolumn{1}{c}{ Áreas } & $\begin{array}{c}\text { Altura } \\
(\mathrm{m})\end{array}$ & $\begin{array}{c}\text { DAP } \\
(\mathrm{cm})\end{array}$ & Forma & $\begin{array}{c}\mathbf{G} \\
\left(\mathrm{m}^{2} \mathrm{ha}^{-1}\right)\end{array}$ & $\begin{array}{c}\mathbf{I} \\
(\text { unid.) }\end{array}$ & $\begin{array}{c}\mathbf{S} \\
\text { (unid.) }\end{array}$ \\
\hline 1- AAA & $5,62 \mathrm{c}$ & $12,42 \mathrm{bc}$ & $2,65 \mathrm{~d}$ & $5,74 \mathrm{c}$ & $3,9 \mathrm{~b}$ & $2,5 \mathrm{~b}$ \\
2- AMA & $7,78 \mathrm{~b}$ & $16,70 \mathrm{a}$ & $3,38 \mathrm{c}$ & $19,93 \mathrm{ab}$ & $7,0 \mathrm{~b}$ & $4,0 \mathrm{~b}$ \\
3- APA - revegetada & $8,35 \mathrm{~b}$ & $9,73 \mathrm{c}$ & $4,15 \mathrm{ab}$ & $12,79 \mathrm{bc}$ & $14,7 \mathrm{a}$ & $7,9 \mathrm{a}$ \\
4- Mata ciliar (MC) & $11,70 \mathrm{a}$ & $14,98 \mathrm{ab}$ & $4,40 \mathrm{a}$ & $29,28 \mathrm{a}$ & $9,7 \mathrm{ab}$ & $4,5 \mathrm{~b}$ \\
5- Reserva legal (RL) & $8,62 \mathrm{~b}$ & $12,12 \mathrm{bc}$ & $4,01 \mathrm{~b}$ & $18,17 \mathrm{abc}$ & $13,1 \mathrm{a}$ & $8,0 \mathrm{a}$ \\
Média & 8,16 & 11,80 & 3,79 & 12,45 & 8,52 & 4,81 \\
CV (\%) & 32,50 & 53,80 & 22,20 & 34,85 & 22,20 & 19,88 \\
Teste F & $15,39 * *$ & $8,21^{* *}$ & $19,41^{* *}$ & $8,31 * *$ & $18,64 * *$ & $18,87 * *$ \\
\hline
\end{tabular}

As médias seguidas da mesma letra não diferem entre si pelo teste Tukey $(\mathrm{p}<0.01)$.

** valores de $\mathrm{F}$ significativos para $\mathrm{P}<0,01$.

Área altamente antropizada (AAA); Área medianamente antropizada (AMA); Área pouco antropizada (APA revegetada).

Para o DAP, as áreas se mostraram estatisticamente semelhantes, destacando a AMA $(0,17 \mathrm{~m})$ e a $\mathrm{MC}(0,15 \mathrm{~m})$. Entretanto, pode-se inferir que, apesar das características diversas das áreas e da semelhança entre os valores do DAP, houve um comportamento análogo entre os indivíduos arbóreos.

Quanto a Forma, os resultados demonstraram que, quando os indivíduos arbóreos encontram-se tutorados ou acompanhados por outros, tendem a apresentar fuste mais ereto e com poucas nuances. Foram observados locais com maiores valores de alturas, maiores índices populacionais, tanto de árvores avaliadas, quanto de não avaliadas e de dossel semifechado a fechado, na APA - revegetada, MC e RL. Em contrapartida, a AAA e AMA foram locais onde os indivíduos arbóreos propiciaram as notas mais baixas.

A MC foi o local que apresentou as maiores alturas e notas da forma, podendo supor a existência de uma correlação positiva entre estes caracteres. Em contrapartida, na AAA e AMA apresentaram os menores valores de número de indivíduos e diversidade de espécies por parcela, evidenciando a influência do ambiente na performance da forma.

As espécies de maior área basal estão localizadas na MC (29,28 $\left.\mathrm{m}^{2} \mathrm{ha}^{-1}\right)$, AMA (19,93 $\mathrm{m}^{2} \mathrm{ha}^{-1}$ ) e RL (18,17 $\mathrm{m}^{2} \mathrm{ha}^{-1}$ ) (Tabela 3). Embora haja semelhança estatística dos valores obtidos para área basal na RL e na AMA, elas diferem quanto ao número de indivíduos e Cultura Agronômica, Ilha Solteira, v.24, n.3, p.327-344, 2015 
diversidade de espécies por parcela. Sendo que, na AMA ocorre metade da quantidade de espécies e aproximadamente metade da quantidade de indivíduos que ocorrem na RL. A presença do gado provavelmente está dificultando a regeneração natural e proporcionando uma pressão de seleção a favor dos indivíduos arbóreos já existentes, apresentando o maior valor do DAP e valores de área basal, estatisticamente iguais aos encontrados na RL e MC. Há que se considerar ainda, o relato de Reis e Kageyama (2003) os quais salientam que, as gramíneas no geral, produzem substâncias capazes de evitar o crescimento de outras espécies, por meio do processo denominado antibiose. Presume-se que haja competição da braquiária o que inibe o desenvolvimento de outras espécies vegetais. Para Durigan et al. (1998) as gramíneas competem com as espécies do cerrado, tornando lento ou até impossibilitando o processo de regeneração.

De acordo com a Resolução n.64 de 10 de setembro de 2009, da Secretaria do Meio Ambiente do Estado de São Paulo, que trata do detalhamento das fisionomias da vegetação de Cerrado e de seus estágios de regeneração, conforme Lei Estadual n.13.550, de 2 de junho de 2009, pode-se inferir que a MC apresentou valores de área basal próximos, mas aquém do indicado nesta resolução, como requisito a ser considerado como fisionomia de Cerradão, ou seja, $20 \mathrm{~m}^{2} \mathrm{ha}^{-1}$. Entretanto, o número de indivíduos por hectare desta fisionomia é de 2200 ind ha ${ }^{-1}$, o que não foi observado em nenhuma área avaliada. Tratando da APA - revegetada (1470 ind ha $\mathrm{ha}^{-1} 12,80 \mathrm{~m}^{2} \mathrm{ha}^{-1}$ ) foi a que mais se aproximou da fisionomia de Cerrado stricto sensu, caracterizado pela mesma resolução, ou seja, projeção da copa das áreas cobrindo cerca de $50 \%$ do terreno, 1500 ind ha $^{-1}$, área basal em torno de $10 \mathrm{~m}^{2} \mathrm{ha}^{-1}$ e altura das árvores de 3 a $6 \mathrm{~m}$, raramente ultrapassando $10 \mathrm{~m}$.

O número de indivíduos (I) na parcela foi maior na APA - revegetada (14,7 ou 1470 ind $\left.\mathrm{ha}^{-1}\right)$ não apresentando diferença estatística em relação à RL (13,1 ou 1310 ind ha $\left.{ }^{-1}\right) \mathrm{e}$ MC (9,7 ou 970 ind ha $^{-1}$ ). Verifica-se que as áreas altamente (AAA) e medianamente (AMA) antropizadas ainda carecem de condições para um estágio mais avançado de regeneração, diferentemente das demais, as quais apresentaram condições que favoreceram a sucessão ecológica e o desempenho das espécies (Tabela 3).

A diversidade de espécies foi maior na RL (8,0 ou 800 esp ha $\left.{ }^{-1}\right)$ e na APA revegetada (7,9 ou 790 esp ha $\left.{ }^{-1}\right)$, inclusive diferindo estatisticamente da MC (4,5 ou 450 esp $\left.\mathrm{ha}^{-1}\right)$, AMA (4,0 ou $\left.400 \mathrm{esp} \mathrm{ha}^{-1}\right)$ e da AAA (2,5 ou 250 esp ha $\left.{ }^{-1}\right)$. A RL representa como era o Cerrado, entretanto, atualmente, podem ser observadas espécies de floresta semidecídua, sinalizando a ocorrência de um ecótono, como constatado por Aragão (2008). Esta área sofre influência causada pela elevação do nível de água do reservatório e fogo. Evidencia-se que estas estimativas de espécies por hectare tenham uma relação direta com as características da área, o que na prática pode não ser efetiva e ainda que, a sucessão ecológica entre as espécies arbóreas nestas áreas possa estar ocorrendo de maneira mais intensa, entre outros, pelo fato de haver uma maior diversidade de espécies. Contudo, verificou-se que a quantidade de espécies nas áreas mais antropizadas corresponde a no máximo $50 \%$ a menos de espécies que uma área onde não ocorreu processo antrópico (Tabela 3).

Cultura Agronômica, Ilha Solteira, v.24, n.3, p.327-344, 2015 
Na AAA foi verificada a menor quantidade de espécies, representando em torno de um terço dos valores obtidos para a APA - revegetada, RL e abaixo do que seria necessário para ser considerado Campo sujo, conforme resolução anteriormente mencionada. Já a área basal, representa menos da metade dos valores encontrados para a APA - revegetada, indicativo de que o número reduzido de espécies e indivíduos por parcela e responsável pela menor área basal.

Outro fator importante que influencia o desenvolvimento das espécies arbóreas está relacionado aos atributos físicos do solo, principalmente em função do crescimento do sistema radicular, conforme descrito por Carvalho et al. (2004) para solos sob sistemas agroflorestais. Segundo Juhász et al. (2006) a característica física dos solos é responsável pela disponibilidade de água, dessa forma, a vegetação predominante, principalmente em áreas de cerradão, mostra-se adaptada para as necessidades hídricas impostas, limitando ou não o desenvolvimento.

Em se tratando dos atributos físicos do solo estudados, observou-se diferenças significativas entre as áreas para todas as profundidades analisadas (Tabela 4). Na profundidade de 0,0 a 0,10 m verificou-se que a macroporosidade foi maior na área de RL, diferindo estatisticamente dos demais e ultrapassando o preconizado por Kiehl (1979) como valor ideal para macroporos $\left(0,17 \mathrm{~m}^{3} \mathrm{~m}^{-3}\right)$. A macroporosidade apresenta importância no fluxo de água no solo e devido sua variabilidade poder ser utilizada como um atributo indicador da condutividade hidráulica (SANTANA et al., 2006).

Assim, o valor obtido para a macroporosidade na AMA encontra-se o menor dentre os demais, sendo um fator que constitui restrição não somente ao fluxo de água, mas ao crescimento e desenvolvimento radicular das espécies vegetais. Por se tratar de área sob pastagem, a diminuição da macroporosidade nesta profundidade pode, provavelmente, ser resultante do pisoteio animal. Esse efeito também é constatado por meio da alta densidade do solo nesta profundidade, apresentando o maior valor para a AMA e AAA, diferindo estatisticamente das demais. Apesar da densidade na área AAA ser estatisticamente igual à AMA, a macroporosidade não apresentou valor abaixo de $0,10 \mathrm{~m}^{3} \mathrm{~m}^{3}$, capaz de comprometer o desenvolvimento do sistema radicular e o desenvolvimento da planta. Comportamento idêntico foi observado por Vzzotto et al. (2000) quando analisaram o efeito do pisoteio bovino sobre os atributos físicos do solo, complementando que 6 meses sem o pisoteio não foram suficientes para que as raízes das gramíneas realizassem a descompactação.

Os resultados de macro e microporosidade nesta profundidade foram responsáveis pela menor porosidade total ( $\mathrm{P}$ total) na AAA e na AMA. Na APA - revegetada, a regeneração de espécies arbóreas, constatada por meio do maior número de indivíduos e de espécies por parcelas, pode ter contribuído para melhorias dos atributos físicos do solo, de forma que, a porosidade total nesta área foi igual estatisticamente às áreas de MC e RL. Silva (2007) avaliando os atributos físicos do solo em reflorestamento ciliar, às margens do reservatório da Usina Hidrelétrica de Ilha Solteira, encontrou valores que refletiram em um melhor estado de recuperação do solo, atribuindo à vegetação implantada e regenerante.

Cultura Agronômica, Ilha Solteira, v.24, n.3, p.327-344, 2015 
Tabela 4. Valores médios de macroporosidade, microporosidade, porosidade total e densidade do solo oriundos das amostras de solo das diferentes áreas avaliadas nas profundidades de $0-0,10 ; 0,10-0,20 ; 0,20-0,30$ e de $0,30-0,40 \mathrm{~m}$.

\begin{tabular}{|c|c|c|c|c|}
\hline Área & $\begin{array}{c}\text { Macro } \\
\ldots\end{array}$ & $\begin{array}{c}\text { Micro } \\
\mathbf{n}^{3} \mathbf{m}^{-3} \ldots . . . .\end{array}$ & P total & $\begin{array}{c}\text { Densidade } \\
\mathrm{kg} \mathrm{dm}^{-3}\end{array}$ \\
\hline \multicolumn{5}{|c|}{$\begin{array}{l}\text { Profundidade } 0-0,10 \mathrm{~m} \\
\end{array}$} \\
\hline AAA & $0,123 \mathrm{bc}$ & $0,273 \mathrm{c}$ & $0,396 \mathrm{~b}$ & $1,46 \mathrm{a}$ \\
\hline AMA & $0,070 \mathrm{c}$ & $0,307 \mathrm{bc}$ & $0,377 \mathrm{~b}$ & $1,54 \mathrm{a}$ \\
\hline APA - revegetada & $0,134 \mathrm{~b}$ & $0,364 \mathrm{a}$ & $0,498 \mathrm{a}$ & $1,25 \mathrm{~b}$ \\
\hline Mata ciliar & $0,114 \mathrm{bc}$ & $0,361 \mathrm{ab}$ & $0,476 \mathrm{a}$ & $1,24 \mathrm{~b}$ \\
\hline Reserva legal & $0,196 \mathrm{a}$ & $0,322 \mathrm{abc}$ & $0,518 \mathrm{a}$ & $1,22 \mathrm{~b}$ \\
\hline Média & 0,13 & 0,31 & 0,44 & 1,36 \\
\hline $\mathrm{CV}(\%)$ & 33,96 & 12,85 & 7,35 & 6,59 \\
\hline Teste F & 5,29 & 7,29 & 22,75 & $14,51 * *$ \\
\hline \multicolumn{5}{|c|}{ Profundidade 0,10-0,20 m } \\
\hline $\mathrm{AAA}$ & $0,125 \mathrm{ab}$ & $0,263 \mathrm{~b}$ & $0,388 \mathrm{c}$ & $1,49 \mathrm{a}$ \\
\hline AMA & $0,113 \mathrm{ab}$ & $0,286 \mathrm{~b}$ & $0,399 \mathrm{bc}$ & $1,50 \mathrm{a}$ \\
\hline APA - revegetada & $0,131 \mathrm{ab}$ & $0,355 \mathrm{a}$ & $0,486 \mathrm{a}$ & $1,29 \mathrm{~b}$ \\
\hline Mata ciliar & $0,077 \mathrm{~b}$ & $0,359 \mathrm{a}$ & $0,436 \mathrm{~b}$ & $1,40 \mathrm{ab}$ \\
\hline Reserva legal & $0,153 \mathrm{a}$ & $0,340 \mathrm{a}$ & $0,494 \mathrm{a}$ & $1,31 \mathrm{~b}$ \\
\hline Média & 0,12 & 0,31 & 0,43 & 1,41 \\
\hline $\mathrm{CV}(\%)$ & 35,06 & 12,35 & 7,74 & 6,85 \\
\hline Teste F & 1,91 & $8,59 * *$ & $14,58 * *$ & \\
\hline \multicolumn{5}{|c|}{ Profundidade $0,20-0,30 \mathrm{~m}$} \\
\hline AAA & $0,112 \mathrm{ab}$ & $0,271 \mathrm{c}$ & $0,383 \mathrm{~b}$ & $1,53 \mathrm{a}$ \\
\hline AMA & $0,098 \mathrm{~b}$ & $0,306 \mathrm{bc}$ & $0,404 \mathrm{~b}$ & $1,48 \mathrm{a}$ \\
\hline APA - revegetada & $0,095 \mathrm{~b}$ & $0,380 \mathrm{a}$ & $0,475 \mathrm{a}$ & $1,33 \mathrm{~b}$ \\
\hline Mata ciliar & $0,044 \mathrm{c}$ & $0,364 \mathrm{a}$ & $0,408 \mathrm{~b}$ & $1,45 \mathrm{a}$ \\
\hline Reserva legal & $0,147 \mathrm{a}$ & $0,347 \mathrm{ab}$ & $0,495 \mathrm{a}$ & $1,30 \mathrm{~b}$ \\
\hline Média & 0,11 & 0,32 & 0,42 & 1,44 \\
\hline $\mathrm{CV}(\%)$ & 27,96 & 11,48 & 8,54 & 5,92 \\
\hline Teste F & 7,39 & $10,02 * *$ & & \\
\hline \multicolumn{5}{|c|}{ Profundidade $0,30-0,40 \mathrm{~m}$} \\
\hline $\mathrm{AAA}$ & $0,086 \mathrm{~b}$ & $0,283 \mathrm{~b}$ & $0,369 \mathrm{c}$ & $1,59 \mathrm{a}$ \\
\hline AMA & $0,136 \mathrm{a}$ & $0,295 \mathrm{~b}$ & $0,430 \mathrm{~b}$ & $1,39 \mathrm{~b}$ \\
\hline $\mathrm{APA}$ - revegetada & $0,113 \mathrm{ab}$ & $0,375 \mathrm{a}$ & $0,488 \mathrm{a}$ & $1,27 \mathrm{c}$ \\
\hline Mata ciliar & $0,043 \mathrm{c}$ & $0,367 \mathrm{a}$ & $0,410 \mathrm{bc}$ & $1,48 \mathrm{~b}$ \\
\hline Reserva legal & $0,137 \mathrm{a}$ & $0,364 \mathrm{a}$ & $0,500 \mathrm{a}$ & $1,28 \mathrm{c}$ \\
\hline Média & 0,1 & 0,32 & 0,42 & 1,45 \\
\hline $\mathrm{CV}(\%)$ & 29,74 & 10,71 & 9,67 & 5,55 \\
\hline Teste F & $9,62 * *$ & $9,63 * *$ & $8,63 * *$ & 12,51 \\
\hline
\end{tabular}

As médias seguidas da mesma letra não diferem entre si pelo teste Tukey $(\mathrm{p}<0.01)$.

** valores de $\mathrm{F}$ significativos para $\mathrm{P}<0,01$.

Área altamente antropizada (AAA); Área medianamente antropizada (AMA); Área pouco antropizada (APA revegetada).

Na profundidade de 0,10 a $0,20 \mathrm{~m}$, na área de RL foi observado o maior valor de macroporosidade e o menor para MC, o que apresentou um decréscimo ao longo das profundidades, evidenciando a antropização ocorrida no passado. Na AMA foi observada 
uma melhora nas condições de macroporosidade com o aumento da profundidade. Este fato é confirmado com os valores de densidade que foram iguais estatisticamente à AAA e AMA, ambas com maior grau de antropização.

A microporosidade apresentou uma estratificação das áreas, sendo diferente estatisticamente com os maiores valores para as áreas de RL, MC e APA - revegetada e menores nas demais. Somente na área de RL, a microporosidade apresentou aumento com a profundidade, devido provavelmente, ao adensamento natural do solo.

Os maiores valores de porosidade total foram observados para as áreas de RL e na APA - revegetada, esse fato deve-se aos menores valores de densidade do solo observados para estas áreas, associado ao fato de serem áreas isoladas (cercadas) e consequentemente, preservadas.

Com relação à densidade no solo, o aumento apresentado para a $\mathrm{MC}$, permitiu que sua condição fosse comparada às apresentadas nas áreas degradadas. Na AMA, mesmo com o maior valor de densidade do solo houve aumento da porosidade total, principalmente em relação a profundidade de 0 a $0,10 \mathrm{~m}$. Em virtude da utilização da área como pastagem, o sistema radicular das gramíneas pode ser o responsável pelo aumento da porosidade nesta profundidade, contribuindo para a formação de bio-poros em profundidade, mesmo que seja aplicada uma carga em superfície com o pisoteio.

Na profundidade de 0,20 a 0,30 m foi observado que na RL e AAA obtiveram os maiores valores para macroporosidade. Nestas duas áreas, os valores de macroporosidade encontram-se acima do limite crítico de $0,10 \mathrm{~m}^{3} \mathrm{~m}^{3}$, preconizado por Greenland (1981) indicando uma boa condição para esse atributo. Quanto às outras áreas, os valores são inferiores e encontram-se abaixo do limite crítico, sendo observada uma redução mais acentuada dessa propriedade na área de MC, que apresentou o menor valor.

As áreas de RL e APA - revegetada apresentaram valores de P total, superiores aos demais, diferindo estatisticamente e vinculados diretamente, com os menores valores de densidade. Apesar das diferenças estatísticas obtidas entre os valores das áreas avaliadas nesta profundidade para $\mathrm{P}$ total, os valores mantiveram-se próximos aos obtidos para as outras profundidades.

A densidade do solo foi maior na AAA, AMA e na MC, refletindo o comportamento da macroporosidade, com exceção para a AAA. Comportamento semelhante ao verificado nas profundidades mais superficiais, indicando a ação das raízes até a profundidade de 0,30 m.

Para a profundidade de 0,30 a 0,40 m, a área de RL, AMA e APA - revegetada apresentaram estatisticamente os maiores valores de macroporosidade e P total. Já para este último atributo, observou-se o mesmo comportamento que vinha sendo apresentado desde a profundidade de 0 a $0,10 \mathrm{~m}$, ou seja, maiores valores e diferentes estatisticamente para a APA - revegetada e RL e menores valores, diferentes estatisticamente para densidade do solo. Estes resultados ratificam o que tende a ocorrer de forma natural no solo, em ambientes preservados.

Cultura Agronômica, Ilha Solteira, v.24, n.3, p.327-344, 2015 
Nas áreas AMA e APA - revegetada, foi observado, em relação a profundidade anterior, um aumento da macroporosidade com o menor valor na MC. Essa condição continua semelhante, desde a profundidade 0 a $0,10 \mathrm{~m}$, abaixo dos valores apresentados para AAA, considerada a área mais degradada.

Os valores de microporosidade foram menores nas AAA e AMA, comportamento análogo ao observado nas outras profundidades. A diminuição da densidade do solo na AMA e APA - revegetada foi verificada com a redução da microporosidade e aumento da macroporosidade, culminando com o aumento da $\mathrm{P}$ total, nesta profundidade.

A porosidade total na APA - revegetada foi igual estatisticamente à RL e apresentaram os maiores valores, diferindo dos demais e refletindo na densidade, com os menores valores, respectivamente.

Nas profundidades avaliadas observaram-se os maiores valores de $\mathrm{P}$ total para as áreas de RL e APA - revegetada. Na AMA houve diminuição da densidade e aumento de porosidade total a medida que se aprofundou as amostragens de solo. Da mesma forma, Alves (1992) avaliando as propriedades físicas e químicas de um Latossolo Roxo sob sistema de rotação de culturas com plantio direto observou em solos degradados, que há relação inversa entre densidade do solo e porosidade total.

Moraes et al. (2002) num estudo da densidade e porosidade do solo como forma a diagnosticar o estado de degradação de solos sob pastagens em região de cerrado, concluíram que, o Latossolo Vermelho sob pastagens de Brachiaria decumbens, quando com densidade superior a $1,30 \mathrm{~kg} \mathrm{dm}^{-3}$ e macroporosidade abaixo de $0,20 \mathrm{~m}^{3} \mathrm{~m}^{-3}$ considerase compactado ou em processo de compactação. Já para Reichert et al. (2003) consideram $1,55 \mathrm{~kg} \mathrm{dm}^{-3}$ como densidade crítica para o bom crescimento do sistema radicular em solos de textura média, sendo a mesma do presente trabalho e que, apenas a AAA na profundidade de 0,30 a 0,40 $\mathrm{m}$ apresentou valor superior a este.

Comportamento inverso para as áreas de MC, APA - revegetada e AAA. Na APA revegetada, o surgimento de espécies arbóreas pode também estar relacionado aos atributos físicos do solo, principalmente em função do crescimento do sistema radicular. Estes benefícios também foram descritos por Carvalho et al. (2004) para solos sob sistemas agroflorestais.

Na Mata ciliar a macroporosidade apresentou decréscimo dos valores com o aumento da profundidade, além de que, os valores de $\mathrm{P}$ total e densidade serem estatisticamente semelhantes aos apresentados para as áreas mais impactadas (AAA e AMA), com exceção da profundidade 0 a $0,10 \mathrm{~m}$. Num estudo do movimento de água e resistência à penetração em Latossolo Vermelho distrófico, Souza e Alves (2003) relataram que a degradação da estrutura do solo ocorre quando há modificações no arranjamento de suas partículas, provocando diminuição no tamanho dos poros, especialmente os macroporos, levando à redução na área da seção transversal para o fluxo de água, juntamente com percursos mais tortuosos para o movimento de fluido, afetando com isso o processo de infiltração. 
Enquanto o solo sob MC impõe condições para o crescimento de espécies arbóreas adaptadas a ciclos de umedecimento e secagem, na AAA e AMA, o desenvolvimento pode estar mais relacionado com as condições edáficas, permitindo o desenvolvimento de um número menor de espécies arbóreas adaptadas às condições adversas em relação aos atributos físicos.

Com base nos valores apresentados para os atributos físicos do solo, entre diferentes níveis de antropização e profundidades, pode-se destacar que o comportamento das espécies arbóreas encontradas na APA - revegetada pode estar sendo beneficiado por estas propriedades, melhor evidenciado pelos elevados valores de microporosidade em todas as profundidades, responsável pela retenção de água.

Destacaram-se seis espécies arbóreas, presentes em quatro das cinco áreas avaliadas, sendo consideradas de interesse quanto à conservação genética in situ (Tabela 2), provavelmente, por estarem mais adaptadas que as demais às condições adversas a que foram submetidas; são elas: Astronium fraxinifolium, Terminalia argentea, Curatella americana, Cupania vernalis, Qualea jundiahy e Andira cuyabensis. Esta constatação denota serem espécies com capacidade de adaptação a condições adversas. Dentre estas, a espécie $C$. americana apresentou sob a projeção de sua copa, em meio a serapilheira, espécies arbóreas regenerantes, na sua grande maioria, frutíferas; contribuindo sobremaneira para a dispersão dos propágulos e regeneração natural da vegetação. São elas: Diospyros híspida; Byrsonima verbacifolia; Byrsonima basiloba; Byrsonima verbacifolia; Brosimum gaudichaudii; Xylopia aromatica; Astronium fraxinifolium; Jacaranda cuspidifolia; Annona crassiflora; Annona pygmaea; Casearia sylvestris; Anacardium humile; Rhamnidium elaeocarpus; Psidium guajava; Salacia elliptica, entre outras.

Com vistas a preservar a biodiversidade, monitorar e conhecer com maiores detalhes a estrutura populacional das espécies arbóreas contidas nas áreas avaliadas sugere-se que sejam criados núcleos de conservação genética in situ em todas as áreas, fornecendo condições de desenvolvimento, inclusive para o banco de sementes e rebrota de raízes. Esta proposta adequa-se à condição de alta diversidade de espécies, constatada no presente trabalho e pelo fato que este tipo de conservação requer longo prazo e várias gerações. Contudo, esta modalidade de conservação não é estática, uma vez que permite interações interespecíficas, sem prejudicar a evolução e coevolução. Entre as espécies, a $C$. americana pode ser considerada como espécie nucleadora, uma vez que, coloniza uma área, sendo uma das primeiras a surgir e facilitar a regeneração de outras espécies sob suas copas.

\section{CONCLUSÃO}

Verificaram-se diferenças na ocupação florestal, diversidade de espécies e desenvolvimento dos indivíduos arbóreos, em função do nível de antropização, estado de conservação das áreas e dos atributos físicos do solo.

Quanto mais isolada a área, maior a ocupação florestal, haja vista a APA - revegetada e a Reserva legal, que apresentaram valores de número de indivíduos e número de espécies,

Cultura Agronômica, Ilha Solteira, v.24, n.3, p.327-344, 2015 
superiores às demais áreas. A Mata ciliar apresentou valores de área basal, altura e forma, superiores às demais áreas. Entretanto, os atributos físicos do solo apresentaram valores inferiores de macroporosidade e elevados de microporosidade, quando comparados às demais áreas.

Em função da presença de vegetação e dos atributos físicos do solo, nas diversas áreas estudadas, seis espécies arbóreas têm potencial adaptativo às condições adversas de solo, sendo indicadas a integrarem programa de conservação genética in situ: Astronium fraxinifolium, Terminalia argentea, Curatella americana, Cupania vernalis, Qualea jundiahy e Andira cuyabensis.

\section{AGRADECIMENTOS}

Os Profs. Titulares Mario Luiz Teixeira de Moraes, Marlene Cristina Alves e Salatiér Buzetti agradecem o Conselho Nacional de Desenvolvimento Científico e Tecnológico $(\mathrm{CNPq})$, pela concessão da bolsa de produtividade.

\section{REFERÊNCIAS BIBLIOGRÁFICAS}

ALVES, M. C. Sistemas de rotação de culturas com plantio direto em Latossolo Roxo: Efeitos nas propriedades físicas e químicas. 1992. $173 \mathrm{f}$. Tese (Doutorado em Solos e Nutrição de Plantas) - Escola Superior de Agricultura "Luiz de Queiroz", Universidade de São Paulo, Piracicaba, 1992.

ALVES, M. C.; SOUZA, Z. M. Recuperação de área degradada por construção de hidroelétrica com adubação verde e corretivo. Revista Brasileira de Ciência do Solo, Viçosa, v. 32, n. 6, p.2505-2516, 2008.

ARAGÃO, S. F. Conservação genética in situ de espécies arbóreas que ocorrem na transição da floresta estacional semidecidual e o Cerrado em Selvíria - MS. 2008. 131 f. Dissertação (Mestrado em Produção Vegetal) - Faculdade de Engenharia de Ilha Solteira, Universidade Estadual Paulista, Ilha Solteira, 2008.

ARAUJO, S. M. V. G. As áreas de preservação permanente e a questão urbana. Brasília: Câmara dos Deputados, 2002. 12 p. (Consultora Legislativa da Área XI Meio Ambiente e Direito Ambiental, Organização Territorial, Desenvolvimento Urbano e Regional).

CARVALHO, R.; GOEDERT, W. J.; ARMANDO M. S. Atributos físicos de um solo sob sistema agroflorestal. Pesquisa Agropecuária Brasileira, Brasília, v. 39, n. 11, p.1153$1155,2004$.

Cultura Agronômica, Ilha Solteira, v.24, n.3, p.327-344, 2015 
DEMATTÊ, J. L. I. Levantamento detalhado dos solos do "Campus experimental de Ilha Solteira”. Piracicaba: Escola Superior Agrícola Luiz de Queiroz, 1980. 119 p.

DURIGAN, G.; CONTIÉRI, W. A.; FRANCO, G. A. D. C.; GARRIDO, M. A. O. Indução do processo de regeneração da vegetação de cerrado em área de pastagem, Assis, SP. Acta Botânica Brasilica, São Paulo, v. 12, n. 3, p.421-429, 1998.

DURIGAN, G., RODRIGUES, R. R.; SCHIAVINI, I. A heterogeneidade ambiental definindo a metodologia de amostragem da floresta ciliar. In: RODRIGUES, R. R.; LEITÃO FILHO, H. F. (eds.). Matas ciliares: conservação e recuperação. São Paulo: EDUSP, 2000. p.159-167.

EITEN, G. Brazilian "Savannas". In: HUNNTLEY, B. J.; WALKER, B. H. Ecology of tropical savanna. Berlin: Heidelberg, 1982. p. 25-47.

EMPRESA BRASILEIRA DE PESQUISA AGROPECUÁRIA - EMBRAPA. Manual de métodos de análise de solo. 2.ed. Rio de Janeiro: Centro Nacional de Pesquisa de Solos, 1997. $212 \mathrm{p}$.

EMPRESA BRASILEIRA DE PESQUISA AGROPECUÁRIA - EMBRAPA. Sistema brasileiro de classificação de solos. 2.ed. Rio de Janeiro: Centro Nacional de Pesquisa de Solos, 2006. 306 p.

GREENLAND, D. J. Soil managment and soil degradation. Journal of Soil Science, London, v. 32, n. 3, p.301-322, 1981.

GUERRA, C. R. S. B. Conservação genética ex situ de populações naturais de Myracrodruon urundeuva Fr. All. em sistema silvipastoril. 2008. 108 f. Tese (Doutorado em Produção Vegetal) - Faculdade de Engenharia de Ilha Solteira, Universidade Estadual Paulista, Ilha Solteira, 2008.

JUHÁSZ, C. E. P.; CURSI, P. R.; COOPER, M.; OLIVEIRA, T. C.; RODRIGUES, R. R. Dinâmica físico-hídrica de uma topossequência de solos sob savana florestada (cerradão) em Assis, SP. Revista Brasileira de Ciência do Solo, Campinas, v. 30, n. 3, p.401-412, 2006.

KAGEYAMA, P.; GANDARA, F. B.; VENCOVSKY, R. Conservação genética in situ de espécies arbóreas tropicais. In: NASS, L. L.; VALOIS, A. C. C.; MELO, I. C.; VALADARES-INGLIS, M. C. (Ed.). Recursos genéticos e melhoramento - Plantas. Rondonópolis: Fundação MT, 2001. p. 149-158.

KIEHL, E. J. Manual de edafologia: relação solo-água-planta. São Paulo: Agronômica Ceres, 1979. $262 \mathrm{p}$.

Cultura Agronômica, Ilha Solteira, v.24, n.3, p.327-344, 2015 
KOPPEN, W. Climatologia. México: Fondo de Cultura Econômica, 1948. 478 p.

MORAES, M. F.; OLIVEIRA, G. C.; KLIEMANN, H. J.; SEVERIANO, E. C.; SARMENTO, P. H. L.; NASCIMENTO, M. O. Densidade e porosidade do solo como diagnóstico do estado de degradação de solos sob pastagens na região dos cerrados. In: SIMPÓSIO NACIONAL SOBRE RECUPERAÇÃO DE ÁREAS DEGRADADAS, 5, 2002, Belo Horizonte. Anais... Belo Horizonte: SOBRADE, 2002. p. 256-258.

MYERS, N.; MITTERMEIER, R. A.; MITTERMEIER, C. G.; FONSECA, G. A. B.; KENT, J. Biodiversity hotspots for conservation priorities. Nature, London, v. 403, n. 12, p.853-858, 2000.

REICHERT, J. M.; REINERT, D. J.; BRAIDA, J. A. Qualidade do solo e sustentabilidade de sistemas agrícolas. Revista Brasileira de Ciências Ambientais, Santa Maria, v. 27, n. 1, p.29-48, 2003.

REIS, A.; BECHARA, F. C.; ESPINDOLA, M. B.; VIERA, N. K.; SOUZA, L. L. Restauração de áreas degradadas: a nucleação como base para incrementar os processos sucessionais. Natureza \& Conservação, Curitiba, v. 1, n. 1, p.28-36, 2003.

REIS, A.; KAGEYAMA, P. Y. Restauração de áreas degradadas utilizando interações interespecíficas. In: KAGEYAMA, P. Y.; OLIVEIRA, R. E.; MORAES, L. F. D.; ENGEL, V. L.; GANDARA, F. B. (Orgs.). Restauração ecológica de ecossistemas naturais. Botucatu: FEPAF, 2003. p. 91-110.

RODRIGUES, G. B.; MALTONI, K. L.; CASSIOLATO, A. M. R. Relação revegetação atributos físicos do solo em uma área degradada de cerrado. In: CONGRESSO BRASILEIRO DE CIÊNCIA DO SOLO, 29, 2003, Ribeirão Preto. Anais... Ribeirão Preto: UNESP, 2003. CD-ROM.

RODRIGUES, G. B.; MALTONI, K. L. Influência da revegetação em atributos químicos de solos de cerrado fortemente impactados. In: CONGRESSO DE INICIAÇÃO CIENTÍFICA DA UNESP, 14. 2002, Presidente Prudente. Anais... Presidente Prudente: UNESP, 2002. CD-ROM.

RODRIGUES, G. B.; MALTONI, K. L.; CASSIOLATO, A. M. R. Dinâmica da regeneração do subsolo de áreas degradadas dentro do bioma Cerrado. Revista Brasileira de Engenharia Agrícola e Ambiental, Campina Grande, v. 11, n. 1, p.73-80, 2007.

SANTANA, M. B.; SOUZA, L. S.; SOUZA, L. D.; FONTES, L. E. F. Atributos físicos do solo e distribuição do sistema radicular de citros como indicadores de horizontes coesos em dois solos de tabuleiros costeiros do Estado da Bahia. Revista Brasileira de Ciência do Solo, Campinas, v. 30, n. 1, p.1-12, 2006. 
SILVA, A. M. Reflorestamento ciliar à margem do reservatório da hidrelétrica de Ilha Solteira em diferentes modelos de plantio. 2007. 137 f. Dissertação (Mestrado em Produção Vegetal) - Faculdade de Engenharia de Ilha Solteira, Universidade Estadual Paulista, Ilha Solteira, 2007.

SIQUEIRA, L. P. Monitoramento de áreas restauradas no interior do estado de São Paulo. Piracicaba, 2002, 128 f. Dissertação (Mestrado em Recursos Florestais Conservação de Ecossistemas Florestais) - Escola Superior de Agricultura "Luiz de Queiroz", Universidade de São Paulo, Piracicaba, 2002.

SOUZA, Z. M.; ALVES, M. C. Movimento de água e resistência à penetração em um Latossolo Vermelho distrófico de Cerrado, sob diferentes usos e manejos. Revista Brasileira de Engenharia Agrícola Ambiental, Campina Grande, v. 7, n. 1, p.18-23, 2003.

STATISTICAL ANALYSIS SYSTEM INSTITUTE - SAS. SAS/STAT procedure guide for personal computers. 9 ed. Cary NC: SAS Inst., 1999. 334 p.

VZZOTTO, V. R.; MARCHEZAN, E.; SEGABINAZZI, T. Efeito do pisoteio bovino em algumas propriedades físicas do solo de várzea. Ciência Rural, Santa Maria, v. 30, n. 6, p.965-969, 2000.

ZORZETTO, R. Mais verde do que imaginávamos. Boletim FAPESP, São Paulo, n. 145, p. $20-25,2008$. 
